\title{
Fungal stress biology: a preface to the Fungal Stress Responses special edition
}

\author{
Drauzio E. N. Rangel(1) - Alene Alder-Rangel • Ekaterina Dadachova • \\ Roger D. Finlay $\cdot$ Martin Kupiec · Jan Dijksterhuis · Gilberto U. L. Braga • \\ Luis M. Corrochano $\cdot$ John E. Hallsworth
}

Received: 19 May 2015 / Revised: 28 May 2015 / Accepted: 29 May 2015 / Published online: 27 June 2015

(C) Springer-Verlag Berlin Heidelberg 2015

\begin{abstract}
There is currently an urgent need to increase global food security, reverse the trends of increasing cancer rates, protect environmental health, and mitigate climate change. Toward these ends, it is imperative to improve soil health and crop productivity, reduce food spoilage, reduce pesticide usage by increasing the use of biological control, optimize bioremediation of polluted sites, and generate energy from sustainable sources such as biofuels. This review focuses on fungi that can help provide solutions to such problems. We discuss key aspects of fungal stress biology in the context of the papers published in this Special Issue of Current Genetics. This area of biology has relevance to pure and applied research on fungal (and indeed other) systems, including biological control of insect pests, roles of saprotrophic fungi in agriculture and forestry, mycotoxin contamination
\end{abstract}

Communicated by D. E. N. Rangel.

This article is part of the Special Issue "Fungal Stress Responses".

D. E. N. Rangel $(\bowtie) \cdot$ A. Alder-Rangel

Instituto de Pesquisa e Desenvolvimento, Universidade do Vale

do Paraíba, São José dos Campos, SP 12244-000, Brazil

e-mail: drauzio@live.com

\section{E. Dadachova}

Department of Radiology and Microbiology and Immunology, Albert Einstein College of Medicine, 1695A Eastchester Road, Bronx, NY 10461, USA

R. D. Finlay

Department of Forest Mycology and Plant Pathology, Uppsala

BioCenter, Swedish University of Agricultural Sciences,

75007 Uppsala, Sweden

M. Kupiec

Department of Molecular Microbiology and Biotechnology,

Tel Aviv University, 69978 Ramat Aviv, Israel of the food-supply chain, optimization of microbial fermentations including those used for bioethanol production, plant pathology, the limits of life on Earth, and astrobiology.

Keywords Acid, alkali, chaotrope, ethanol, heat, hypoxic, osmotic, and salt stress · Aspergillus wentii $\cdot$ Astrobiology $\cdot$ Beauveria bassiana $\cdot$ Biofuels . Cochliobolus heterostrophus · Compatible solutes . Cryomyces antarcticus $\cdot$ Entomopathogenic fungi . Erythritol and mannitol $\cdot$ Fusarium graminearum $\cdot$ Hortaea werneckii $\cdot$ Metarhizium robertsii $\cdot$ Neurospora crassa . Saccharomyces cerevisiae $\cdot$ Trehalose $\cdot$ Trichoderma atroviride $\cdot \mathrm{UV}-\mathrm{B}$ radiation tolerance

\section{Introduction}

This Special Issue Fungal Stress Responses is dedicated to understanding how fungi, important in natural and

\author{
J. Dijksterhuis \\ CBS-KNAW Fungal Biodiversity Centre, Uppsalalaan 8, \\ 3584, CT, Utrecht, The Netherlands
}

\section{G. U. L. Braga}

Faculdade de Ciências Farmacêuticas de Ribeirão Preto,

Universidade de São Paulo, Ribeirão Preto, SP 14040-903, Brazil

\section{M. Corrochano}

Departamento de Genética, Facultad de Biología, Universidad de Sevilla, Avenida Reina Mercedes 6, Apartado 1095,

41080 Seville, Spain

J. E. Hallsworth

Institute for Global Food Security, School of Biological

Sciences, Queen's University Belfast, MBC, 97 Lisburn Road,

Belfast BT9 7BL, UK 
industrial systems, and for fields ranging from agriculture to astrobiology, can sense and cope with stress. Due to their ubiquitous nature and their extreme variation in growth phenotype, metabolite production, and response(s) to the environment, fungi have the potential to mitigate some of the world's most pressing problems. Fungi play central roles in agriculture, biotechnology, and basic research: they produce molecules used in the pharmaceutical, energy-producing, and food industries, and play important ecological roles. Because most of these applications impose complex and dynamic challenges and stresses upon the fungal cell, it is imperative to elucidate the stress biology of fungi. Furthermore, this can lead to knowledge-based approaches to manipulate and optimize processes driven by these microbes.

The papers in this Special Issue cover a wide range of organisms, both in their natural habitats and in industrial or other anthropogenic systems, such as agriculture. Collectively, these studies relate fungal ecology, cell biology, biophysical mechanisms, and molecular and physiological responses to stress. Here, we summarize the main outline of these articles.

\section{Topics covered in this special issue}

Insect-pathogenic fungi are used as model systems that not only have special significance for biological control, but are also pertinent to other aspects of fungal stress biology. Ortiz-Urquiza and Keyhani (2014) explain that the concept of stress has been rather poorly defined, and that related terminology may be used to refer to any/all condition(s) that differ from the optimum. This might imply that every change in condition can be interpreted as "stressful", and as numerous parameters are in a constant state of flux, organisms are continuously exposed to stress. During infection of the insect host, several, more-profound stresses affect the fungal cells. The authors discuss both the fungi Beauveria bassiana and Metarhizium robertsii, and address the biochemical pathways during a number of stress responses including water loss, reactive oxygen species, and UV radiation. For both species, responses to these stresses include the accumulation of compatible solutes, enzymatic and non-enzymatic scavenging of reactive oxygen species, pigment synthesis, and the induction of DNA-damage repair systems. Many examples are given of molecules involved in signaling from membrane proteins and receptors (e.g., G-proteins and tetraspanins) toward downstream signaling pathways (such as MAP kinase cascades and cAMP-PKA pathways), ultimately leading to the transcription factors that activate genes. Regulation of the stress response during infection is a sensitive and intimate process, as demonstrated by the observation that an adenylate cyclase deletion mutant of $M$. robertsii has only a slightly impaired virulence, while the gene disruption of the downstream PKA catalytic subunit results in a reduction of virulence of $90 \%$. In addition, cross talk of different signaling pathways is observed in B. bassiana, as deletion mutants of the Slt2 MAP kinase pathway (involved in cell wall sensing) resulted in reduced phosphorylation of the transcription factor of another pathway, namely Hog1 (involved in osmotic stress response). The authors conclude that the intricacies of signaling processes (e.g., the roles of epigenetic mechanisms) require more extensive study.

The genus Metarhizium includes remarkably versatile fungi that live as saprotrophs, in association with plants (e.g., as growth promoters), and as organisms that are pathogenic to insects. In their article, "Stress is the rule rather than the exception for Metarhizium", Lovett and St. Leger (2014) also detail an example of transfer of nitrogen from insect cadavers to plants, a dramatic illustration of the intricate ecological networks that occur in soil. The authors follow the infection process and discuss the role of a large number of important molecular factors. For instance, the adhesin MAD1 and hydrophobins during adherence of a perilipin that regulates the turgor pressure of infection structures in the hemocoel is discussed. They highlight the stresses encountered during infection, including nutrient stresses experienced on the cuticle, oxidative stress within the cuticle, and osmotic stress within the hemocoel. Interestingly, it is upon emergence from the host that fungi face what is arguably the most formidable stress; i.e., UV exposure. This is also one of the key environmental factors which limit the effectiveness of these fungi as biological control agents. The authors state that many stress-related genes of Metarhizium have a pleiotropic nature, probably related to the battery of different environmental inputs that the fungus has to respond to. Genetic engineering has the potential, therefore, to increase the stress tolerance of this fungus.

Eleutherio et al. (2014) provide an authoritative review regarding the synthesis and breakdown of trehalose in $S$. cerevisae and applications of trehalose metabolism in yeast and other organisms. They explain what we know and, importantly, what we might still need to discover by placing current knowledge in the context of scientific developments throughout the history of this field. The storyline explores the idiosyncratic character and behavior of this disaccharide and its roles in the protection against multiple stresses. A variety of stress-protection mechanisms are discussed, including water replacement during membrane desiccation, glass formation, and the preferential exclusion hypothesis. In a recent review focusing on biofuel fermentation (Cray et al. 2015), Eleutherio and collaborators also explore the role of kosmotropic activity, a key activity related to the modes-of-action of other compatible solutes (see below; Cray et al. 2013; Ball and Hallsworth 
2015). The molecular biology of trehalose synthesis (common to many types of living system) and its regulation are examined in detail, with a focus on the TPS/TPP pathway in $S$. cerevisiae. As the authors explain, trehalose transport across the plasma membrane is key to effective stress protection, because trehalose must be located on both sides of the bilayer, and yet the mechanism(s) involved in the presumed re-assimilation of extracellular trehalose are yet to be elucidated. Hydrolysis of trehalose can provide the cell with the energy required to recover from stress. In addition, Eleutherio et al. (2014) provide intriguing insights into the role of trehalose metabolism in the biology of host infection by pathogenic microbes, and they allude to a variety of therapies to control infectious diseases.

It is likely that the capacity of fungal cells to sense and respond to visible light originated as a mechanism of defense against the consequences of harmful UV radiation and other damage that can be inflicted by excessive exposure. Biological clocks that run according to a daily cycle (circadian clocks) allow cells to anticipate the presence of light and prepare for daily changes in light and temperature. Fungi use light as a signal from the environment to regulate pigment biosynthesis, development, and entrain the circadian clock. The ascomycete Neurospora crassa has been used as a model for fungal photobiology and clock regulation. The key component of $N$. crassa photoreception is the white collar complex (WCC), a light-responsive transcription factor complex, but the genome contains genes for other types of photoreceptors. They include another type of blue light photoreceptor (cryptochrome), an opsin that is similar to the vertebrate photoreceptors, and two red light photoreceptors (phytochromes). The surge in fungal genome-sequencing projects has catalyzed the identification of fungal photoreceptors in many other species and their role in the photobiology of fungi is being actively investigated. Fuller et al. (2014) summarize in their review "Fungal photobiology: visible light as a signal for stress, space and time" our current knowledge on fungal photobiology with a detailed description of the role of the different types of photoreceptors in $N$. crassa and other fungi. The discovery that some pathogenic fungi use light as a signal during pathogenesis suggests new avenues of research to understand the mechanism of environmental sensing by fungal pathogens and new approaches to treat diseases using photoreceptors as antifungal targets.

Further insights into the stress biology of insect-pathogenic fungi are provided by Huarte-Bonnet et al. (2014), who home in on one stage of infection. "Oxidative stress in entomopathogenic fungi grown on insect-like hydrocarbons" studies the growth of $B$. bassiana on media containing hydrocarbons that feed the fungus when it is on the cuticle of insects. This imposes very specific stresses on the fungi, which now have to deal with reactive oxygen species that must be degraded effectively within the cytoplasm as well as inside peroxisomes. Huarte-Bonnet et al. observe increases in superoxide dismutase and catalase levels when the fungus is inoculated onto hydrocarbon-supplemented media. This was confirmed by qRT-PCR measurements, which detected a substantial increase in expression levels for the $\operatorname{sodl}$, catA, catB, and catP genes. Glutathione peroxidase was also induced, suggesting the removal of peroxides by cooperation between different enzymes. HuarteBonnet et al. provide a lucid, detailed discussion relating to the consequences of growth on cuticle hydrocarbons including the role of cytochrome P450 enzymes (CYP).

The cellular responses to osmotic stress in the yeast $S$. cerevisiae provide the focus for the review of the review by Solé et al. (2014). The key regulator of the response to osmotic stress is the protein kinase Hog1 (homologous to p38 in other organisms), and the authors review the role of this protein during the transcriptional changes that occur during the response to osmotic stress. One of the consequences of osmotic stress is a delay in the cell cycle, which provides time to adapt to the stress before resuming growth. Hog1 modulates the cell cycle arrest after osmotic stress by phosphorylation of several regulators of the cell cycle. A new layer of regulation to the cellular response to stress was introduced with the discovery of 200 long non-coding RNAs (lncRNAs) that are strongly and rapidly induced upon osmotic stress. About 90 of these lncRNAs required $\mathrm{Hog} 1$, and the authors focused on the regulation of one of them, the lncRNA that is complementary to the CDC28 gene, encoding the main cyclin-dependent kinase (CDK) of yeast. Cdc28 regulates the cell cycle, and the authors found that the IncRNA participates in the induction of $C D C 28$ expression to facilitate the re-entry of the cell into the cell cycle after stress. The regulation occurs through a looping mechanism that allows the relocalization of Hog 1 in the promoter of $C D C 28$ to promote additional protein interactions that result in the activation of transcription. Hog 1 homologs may operate through similar mechanisms for transcriptional regulation in other fungi.

The accumulation of pigments may provide protection against excessive visible light, UV radiation, and oxidative stress. Carotenoids are terpenoids with antioxidant properties and have a wide absorption in the visible part of the light spectrum. The accumulation of various types of carotenoids, in many cases after exposure to light, suggests that these pigments may protect the fungal cell from damage caused by excessive illumination. The review by Avalos and Limón (2014) contains a comprehensive analysis of our current knowledge on the biosynthesis and roles of carotenoids and derivatives, in the context of fungal biology. The authors describe the chemical properties and roles of the carotenoids that accumulate in several fungal species, the enzymes that participate in their biosynthesis, and 
their regulation. Carotenoid derivatives, such as trisporic acids and retina, play relevant roles in certain fungi and act as sexual hormones or chromophores.

Medina et al. (2014) examined the effects of high solute concentrations (i.e., low water activity) and temperature on the accumulation of compatible solutes and expression of genes involved in sugar transport and mycotoxin production for xerotolerant/xerophilic fungi. Their paper reports a systems-inspired approach based on quantification of fungal growth rates, intracellular compatible solutes, mycotoxin concentrations, gene expression for key genes involved in sugar transport and mycotoxin production, and creation of a model that can be used to predict conditions under which production of mycotoxins occurs. The solute stressors studied were $\mathrm{NaCl}$, which is an ionic osmotic stressor, and glycerol, which reduces water activity (and is chaotropic at high concentrations) and yet does not impose osmotic stress (see Alves et al. 2015 in this Special Issue). Medina et al. focus on interactions between temperature and stressor concentration as determinants for aspects of fungal growth phenotype and physiology in Aspergillus, Penicillium, and Fusarium. Interestingly, they report a link between up-regulation of sugar transport genes, stress tolerance, and mycotoxin production, which is consistent with both the high energy requirement for tolerance of extreme and multiple stresses, and studies suggesting that the robust stress biology of Aspergillus species is linked to an enhanced primary metabolism, i.e., increased flux in glycolysis and the TCA cycle (Flipphi et al. 2009). The findings of Medina et al. provide key insights needed to understand the high competitive ability of fungi such as Aspergillus species, which can dominate their favored habitats (Cray et al. 2013) and can also be used by agriculturalists and the food industry to prevent mycotoxin contamination of foods and feeds. Furthermore, strains of A. flavus that have deletions in genes involved in the mycotoxin synthesis are being successfully used to competitively exclude toxigenic strains and reduce aflatoxin contamination of peanuts, maize, and cotton (Ehrlich et al. 2015).

In their article "Rock black fungi: excellence in the extremes, from Antarctic to space", Selbmann et al. (2014) review the biology and phylogeny of rock-dwelling black meristematic fungi that can survive at the interface between biology and physics. Such organisms afford insights into early terrestrial life on Earth that was associated with mineral and saline environments. The focus is on diversity, taxonomy, and stress tolerance of black meristematic fungi in the Antarctic, a continent where life "more than on any other continent, is dominated by microbes". These fungi, located mainly in two orders of the Ascomycota, are variously known as black meristematic fungi, black fungi, black yeasts (when inhabiting rocks), and microcolonial or rock-inhabiting fungi. As Selbmann et al. explain, black meristematic fungi are typically polyextremotolerant, rather than extremophilic; their high melanin content gives rise to their exceptional resistance to UV. Selbmann et al. also discuss the link between high melanin levels in black meristematic fungi and efficient retention of the compatible solute glycerol; many other extremely stress-tolerant microbes also have other special adaptations of compatible solute metabolism which contribute to their robust biology. The authors predict a resurgence of research activity into the biology of black meristematic fungi, now that genome sequencing enables the use of various '-omics' (and systems) approaches. This is indeed likely, given that the genome sequences of species such as Hortaea werneckii and Cryomyces antarcticus have recently been published. Furthermore, there is an urgent need to understand these charismatic microbes before climate change endangers their primary habitat.

The paper by Shalaby and Horwitz (2014) reviews the possible role of plant phenolic compounds as signals influencing fungal responses to oxidative stress during interactions with plants. The mode-of-action of some phenolic compounds is known to be mediated by their chaotropic activities (Cray et al. 2014, 2015). Fungi interacting with plants perceive a range of different plant compounds: phenolics and related compounds are ubiquitous in plant cell walls and likely to be encountered by invading pathogens. The authors review responses to different pre-formed and induced phenolics in an attempt to establish connections between studies of signaling and metabolism. Fungal pathogens may metabolize phenolic compounds or respond to them as signals, or both, and the authors suggest that fungal responses to phenolics and oxidants are integrated. Features of the molecular cross talk between plants and prokaryotic pathogens or symbionts are discussed including the possibility of specific recognition of different classes of phenolic compounds in interactions involving Agrobacterium tumefaciens VirA. Evidence from prokaryotic systems is discussed in relation to understanding of recognition of phenols by fungi, in particular in studies of the pathogens Cochliobolus heterostrophus and Fusarium graminearum. Phenolics may provide either antioxidant or pro-oxidant signals to modulate secondary metabolite production, and the authors suggest that better understanding of how fungal pathogens detect plant phenolic compounds as stress signals may facilitate the development of new strategies for control of fungal plant pathogens.

Mechanical damage to microbial cells can break the integrity of the cellular membrane and cause lethality. Whereas all living systems may have developed ways to cope with mechanical injuries, most research has focused on the mechanisms of response to injury in plants and animals. Hyphal breakage upon mechanical injury was previously characterized in fungi (Leonard and Dick 1973) and 
the discovery that injuries promote the activation of asexual development in the ascomycete Trichoderma atroviride has enabled the elucidation of the molecular mechanism of this phenomenon using a combination of genetics and genomics. The review by Hernández-Oñate and HerreraEstrella (2015) summarizes our current knowledge of the mechanisms of the response to injury in $T$. atroviride with the participation of calcium, the production of reactive oxygen species, the regulatory network of MAP kinases, and the lipid signaling pathways. The observations that several of the signaling molecules and the mechanisms that mediate the response to cellular damage are conserved across plant, animal, and fungal species suggest an ancient origin. The authors propose that fungi could be used as models to understand the mechanism of response to mechanical injuries in other types of eukaryotic cells.

The paper by Hohmann (2015) reviews recent progress in understanding osmoregulation in eukaryotes, concentrating in particular on the different roles of Hog1 in glycerol accumulation and volume recovery in yeast. Osmoregulation is critical for cell shape and turgor as well as intracellular dynamics. Glycerol at high concentrations can act as a stressor (Hallsworth et al. 1998; Williams and Hallsworth 2009; Alves et al. 2015) and is used as a compatible solute, playing diverse roles in yeast physiology. The author summarizes different aspects of the HOG MAPK signaling pathway, including the $\operatorname{Sin} 1$ branch and the Sho1 branch that appear to function independently and have different roles. The author reviews studies of how the HOG MAPK pathway communicates with other yeast MAPK pathways to control cell morphogenesis and how cross talk between the MAPK pathways has recently been used to re-wire osmostress-controlled expression of glycerol biosynthesis genes from Hog1 to Kss1-Fus3. The re-routed osmoregulation system offers opportunities to study osmoadaptation in yeast in the absence of the Hog 1 kinase and may prove a useful tool to elucidate Hog1-dependent and independent mechanisms as well as redundancy and robustness of the MAPK system. However, the author also points out that we still lack a systems-level understanding of the interaction of the diverse MAPK signaling pathways in controlling homeostasis and morphogenesis, especially under nonstress conditions, and that many details of the Fps1 glycerol channel are still lacking.

The article entitled "Stress tolerance and virulence of insect-pathogenic fungi are determined by environmental conditions during conidial formation" by Rangel et al. (2015) focuses on the ways the conidial tolerance of entomopathogenic fungi to heat and UV-B radiation is influenced by environmental factors. The data demonstrates that physical, chemical, and nutritional conditions, which are present during mycelial growth, result in pronounced differences in the way the conidia subsequently survive stressful conditions.
The evidence comes from the investigation of the stress phenotypes of Metarhizium robertsii, which was grown on complex medium versus minimal medium. When conidia were produced on minimal medium, it was able to germinate more efficiently and was more resistant to heat and more virulent to insects than when grown on complex medium (see also Rangel et al. 2006a, 2008a, b). Among the conditions that induced higher tolerance to heat or UV-B radiation were the lack of water content and high alkalinity. However, this resistance and the associated robust phenotype come at a price of low conidial yield. Rangel et al. (2015) give insights into the physicochemical properties and ecophysiological roles of erythritol, trehalose, and mannitol in relation to fungal cell biology (see also Alves et al. 2015; Rangel et al. 2006a, b, 2008a, b). The authors have also shown that three levels of phenotypic plasticity occur for stress tolerance exhibited by $M$. robertsii, and this has profound consequences for its stress biology, virulence, and ecology. The authors concluded that physical and chemical parameters in combination with the nutritional factors can result in the significant difference in the conidial response to stress and can be exploited in the production of the entomopathogenic fungi for biological control.

The article by Braga et al. (2015) provides a clear explanation of the interactions between fungal conidia and solar radiation. Conidia are extremely important for the environmental persistence of the filamentous fungi, as well as for host recognition and infection of the pathogenic species of fungi. As both solar UV-A and UV-B can kill the conidia, as well as limit the proliferation and dispersion of fungi, it is important to summarize the available knowledge of the effects of solar radiation on conidia and on the major systems involved in the protection and repair of damage induced by solar UV radiation. The authors also assure the importance of the fungal pigmentation on conidial UV tolerance as published elsewhere (Braga et al. 2006; Rangel et al. 2006b). The review demonstrated that it is possible to manipulate fungi in laboratory conditions so that the resulting strains produce conidia that are more tolerant to solar radiation than wild type.

The article by Fernandes et al. (2015) covers aspects of UV radiation from sunlight, which is the most detrimental environmental factor that affects the viability of entomopathogenic fungi applied for pest control. The outcomes of studies on this topic can be affected by two compounding factors: the sensitivity of most entomopathogenic fungi to UV radiation and the variability of the laboratory methods that are used to study the UV sensitivity of entomopathogenic fungi. The review carefully analyzes several studies that have applied different methods for testing of natural and enhanced susceptibility to UV and makes inter- and intra-species comparisons. Both inter- and intra-species variability probably result from 
the adaptation(s) of individual strains to diverse natural habitats/sets of conditions. Thus, the selection of strains with the best inherent tolerance to UV is an important step to select the most promising entomopathogenic fungi as biological control agents. Isolate screening for better tolerance to UV radiation as documented by Fernandes et al. (2015) as well as screening for superior tolerance to heat and higher virulence as documented elsewhere (Alston et al. 2005; Rangel et al. 2005, 2010; Fernandes et al. 2008, 2009, 2010, 2011) are important for the successful biological control of insects in agriculture. Although the use of UV lamps in the laboratory does not represent the complexity of the field tests, it does provide a useful technique to help find ways to increase the resistance to UV radiation of entomopathogenic fungi, which will eventually undergo the ultimate tests under field conditions.

The paper by (Hillmann et al. 2015) reviews recent studies of metabolic changes in filamentous fungi during adaptation to hypoxia. A better understanding of these changes and how they are regulated is important, because recent research suggests that adaptation to hypoxia is an important virulence trait of pathogenic fungi. The authors discuss studies of model organisms in the genus Aspergillus and suggest that most species have the capacity to grow in atmospheres with sparse amounts of oxygen, but none grows in the complete absence of oxygen. Recent '-omics' studies designed to elucidate the metabolic and energetic consequences of oxygen limitation are discussed with regard to their relevance in clinical and biotechnological applications. Hypoxic conditions can frequently be found in flooded or waterlogged natural environments or in necrotic tissue with oxygen supply disrupted via the blood stream. Transcriptomic and proteomic studies have facilitated the study of fungal responses to low oxygen levels, but due to the high diversity in the hypoxic stress response of filamentous fungi, it is not easy to obtain a common picture of low oxygen responses in all filamentous fungi. In general, genes involved in glycolysis, steroid, and cell wall biosynthesis, as well as secondary metabolite metabolism, seem to be up-regulated, whereas transcript levels of ribosomal and purine/pyrimidine biosynthesis genes are downregulated. This suggests that glycolysis contributes to a larger proportion of ATP generation and that the cell wall undergoes a significant restructuring under hypoxia. The authors conclude that, although we have only just begun to understand which measures filamentous fungi take to thrive in less oxygenated areas, and there are several species-specific aspects in hypoxia adaptation, nitric oxide (NO), and reactive oxygen species, mitochondrial signaling marks at least one common ground among fungal species in the genus Aspergillus.
The article by Alves et al. (2015) examines the biology of solute-induced stresses in the mycotoxin-producing, xerophilic fungus Aspergillus wentii via a series of experiments that includes characterization of growth and compatible solute responses to solute concentration, water activity, and chao-/kosmotropicity for 12 solute stressors. Additional, complementary studies of solute-induced perturbations of macromolecular model systems were carried out, and S. cerevisiae strains/mutants were used to determine chaotropic and osmotic activities of solute stressors. Alves et al. thereby disentangle the various stress parameters by which diverse solutes impact the cellular system, including biologically and biotechnologically pertinent substances such as salts, glucose, polyols, ethanol, and urea. According to the solute interactions with the fungal cell, and their primary modes-of-inhibitory action, these stressors partitioned into a number of natural categories: osmotically active ( $\mathrm{NaCl}, \mathrm{KCl}$, glucose, sorbitol), nonosmotic and weakly to moderately chaotropic (ethanol, urea, ethylene glycol), both highly chaotropic and osmotically active $\left(\mathrm{NH}_{4} \mathrm{NO}_{3}, \mathrm{MgCl}_{2}\right.$, guanidine hydrochloride, $\mathrm{CaCl}_{2}$ ), and inhibitory primarily due to low water activity (glycerol). This study thereby revealed that some stressors, including $\mathrm{MgCl}_{2}$, can inhibit metabolism and cell multiplication via their chaotropic activity and by simultaneously imposing osmotic stress; a finding that is unprecedented for any type of living system. While not an osmotic stressor, it seems likely that ethanol can (at high concentrations) impose simultaneous chaotropicity and low water activityinduced stresses in S. cerevisiae (Hallsworth 1998; Cray et al. 2015). Conversely, high concentrations of $\mathrm{MgCl}_{2}$ may ultimately inhibit the multiplication of extremely halophilic bacteria and Archaea due to chaotropicity rather than water activity or osmotic stress (Hallsworth et al. 2007; Yakimov et al. 2015). Based on their findings, Alves et al. justifiably question the validity of the 'polyextremophile' concept as it is currently applied. Their study also reveals that compatible solutes define the biotic window for fungal growth and metabolism under chaotropic osmotic conditions as well as other solute-induced stresses, and that kosmotropicity activities of compatible solutes can mitigate chaotropicity. The authors also discuss implications for preventing contamination of both the food-supply chain with mycotoxins and life-support systems used during space exploration missions.

Acknowledgments The Universidade do Vale do Paraíba in São José dos Campos, SP, Brazil hosted the International Symposium on Fungal Stress (ISFUS) in October 2014 organized by Drauzio E. N. Rangel, Alene Alder-Rangel, Luis M. Corrochano, and John E. Hallsworth with support from São Paulo Research Foundation (FAPESP). This special edition on Fungal Stress Responses published in Current Genetics was inspired by the International Symposium on Fungal Stress and invited by Dr. Stefan Hohmann, former Editor-in-Chief of 
Current Genetics. This work was supported by grants of the Brazilian National Council for Scientific and Technological Development (CNPq) 473104/2008-3, 478899/2010-6, PQ2 302312/2011-0, and PQ1D 308436/2014-8 for D. E. N. R. This review article was supported by a grant from São Paulo Research Foundation (FAPESP) of Brazil \#2010/06374-1, 2013/50518-6 for D. E. N. R. and 2012/15204-8 for G. U. L. B. This review article was supported in part by a grant from FAPESP \# 2014/01229-4. This work was also supported by European funds (European Regional Development Fund, ERDF), and the Spanish Ministerio de Educación y Ciencia (BIO2012-38520) for L. M. C.

\section{References}

Alston DG, Rangel DEN, Lacey LA, Golez HG, Kim JJ, Roberts DW (2005) Evaluation of novel fungal and nematode isolates for control of Conotrachelus nenuphar (Coleoptera: Curculionidae) larvae. Biol Control 35:163-171

Alves FL, Stevenson A, Baxter E, Gillion JLM, Hejazi F, Hayes S, Morrison IEG, Prior BA, McGenity TJ, Rangel DEN, Magan N, Timmis $\mathrm{KN}$, Hallsworth JE (2015) Concomitant osmotic and chaotropicityinduced stresses in Aspergillus wentii: compatible solutes determine the biotic window. Curr Genet. doi:10.1007/s00294-015-0496-8

Avalos J, Limón MC (2014) Biological roles of fungal carotenoids. Curr Genet. doi:10.1007/s00294-014-0454-X

Ball P, Hallsworth JE (2015) Water structure and chaotropicity: their uses, abuses and biological implications. Phys Chem Chem Phys 17:8297-8305. doi:10.1039/c4cp04564e

Braga GUL, Rangel DEN, Flint SD, Anderson AJ, Roberts DW (2006) Conidial pigmentation is important to tolerance against solar-simulated radiation in the entomopathogenic fungus Metarhizium anisopliae. Photochem Photobiol 82:418-422

Braga GUL, Rangel DEN, Fernandes EKK, Flint SD, Roberts DW (2015) Molecular and physiological effects of environmental UV radiation on fungal conidia. Curr Genet. doi:10.1007/ s00294-015-0483-0

Cray JA, Bell ANW, Bhaganna P, Mswaka AY, Timson DJ, Hallsworth JE (2013) The biology of habitat dominance; can microbes behave as weeds? Microb Biotechnol 6:453-492. doi:10.1111/1751-7915.12027

Cray JA, Bhaganna P, Singhal RS, Patil SV, Saha D, Chakraborty R, Iwaguchi S, Timson DJ, Hallsworth JE (2014) Chaotropic and hydrophobic stress mechanisms of antifungal substances. In: Dehne HW et al (eds) Modern fungicides and antifungal compounds, vol VII. Deutsche Phytomedizinische Gesellschaft, Braunschweig, pp 73-80

Cray JA, Stevenson A, Ball P, Bankar SB, Eleutherio ECA, Ezeji TC, Singhal RS, Thevelein JM, Timson DJ, Hallsworth JE (2015) Chaotropicity: a key factor in product tolerance of biofuel-producing microorganisms. Curr Opin Biotechnol 33:228-259. doi:10.1016/j.copbio.2015.02.010

Ehrlich KC, Moore GG, Mellon JE, Bhatnagar D (2015) Challenges facing the biological control strategy for eliminating aflatoxin contamination. World Mycotoxin J 8:225-233. doi:10.3920/ wmj2014.1696

Eleutherio E, Panek AD, de Mesquita JF, Trevisol E, Magalhães R (2014) Revisiting yeast trehalose metabolism. Curr Genet. doi:10.1007/s00294-014-0450-1

Fernandes EKK, Rangel DEN, Moraes AML, Bittencourt VREP, Roberts DW (2008) Cold activity of Beauveria and Metarhizium, and thermotolerance of Beauveria. J Invertebr Pathol 98:69-78

Fernandes EKK, Moraes AML, Pacheco RS, Rangel DEN, Miller MP, Bittencourt VREP, Roberts DW (2009) Genetic diversity among Brazilian isolates of Beauveria bassiana: comparisons with
non-Brazilian isolates and other Beauveria species. J Appl Microbiol 107:760-774

Fernandes EKK, Keyser CA, Chong JP, Rangel DEN, Miller MP, Roberts DW (2010) Characterization of Metarhizium species and varieties based on molecular analysis, heat tolerance and cold activity. J Appl Microbiol 108:115-128

Fernandes EKK, Angelo IC, Rangel DEN, Bahiense TC, Moraes AM, Roberts DW, Bittencourt VR (2011) An intensive search for promising fungal biological control agents of ticks, particularly Rhipicephalus microplus. Vet Parasitol 182:307-318. doi:10.1016/j.vetpar.2011.05.046

Fernandes EKK, Rangel DEN, Braga GUL, Roberts DW (2015) Tolerance of entomopathogenic fungi to ultraviolet radiation: a review on screening of strains and their formulation. Curr Genet. doi:10.1007/s00294-015-0492-z

Flipphi M, Sun JB, Robellet X, Karaffa L, Fekete E, Zeng AP, Lubicek CP (2009) Biodiversity and evolution of primary carbon metabolism in Aspergillus nidulans and other Aspergillus spp. Fungal Genet Biol 46:S19-S44. doi:10.1016/j.fgb.2008.07.018

Fuller K, Loros J, Dunlap J (2014) Fungal photobiology: visible light as a signal for stress, space and time. Curr Genet. doi:10.1007/ s00294-014-0451-0

Hallsworth JE (1998) Ethanol-induced water stress in yeast. J Ferment Bioeng 85:125-137. doi:10.1016/s0922-338x(97)86756-6

Hallsworth JE, Nomura Y, Iwahara M (1998) Ethanol-induced water stress and fungal growth. J Ferment Bioeng 86:451-456. doi:10.1016/S0922-338X(98)80150-5

Hallsworth JE, Yakimov MM, Golyshin PN, Gillion JLM, D'Auria G, Alves FdL, La Cono V, Genovese M, McKew BA, Hayes SL, Harris G, Giuliano L, Timmis KN, McGenity TJ (2007) Limits of life in $\mathrm{MgCl} 2$-containing environments: chaotropicity defines the window. Environ Microbiol 9:801-813. doi:10.1111/j.1462-2920.2006.01212.x

Hernández-Oñate MA, Herrera-Estrella A (2015) Damage response involves mechanisms conserved across plants, animals and fungi. Curr Genet. doi:10.1007/s00294-014-0467-5

Hillmann F, Shekhova E, Kniemeyer O (2015) Insights into the cellular responses to hypoxia in filamentous fungi. Curr Genet. doi: 10.1007/s00294-015-0487-9

Hohmann S (2015) An integrated view on a eukaryotic osmoregulation system. Curr Genet. doi:10.1007/s00294-015-0475-0

Huarte-Bonnet C, Juárez MP, Pedrini N (2014) Oxidative stress in entomopathogenic fungi grown on insect-like hydrocarbons. Curr Genet. doi:10.1007/s00294-014-0452-z

Leonard TJ, Dick S (1973) Induction of haploid fruiting by mechanical injury in Schizophyllum commune. Mycologia 65:809-822. doi: $10.2307 / 3758520$

Lovett B, St. Leger R (2014) Stress is the rule rather than the exception for Metarhizium. Curr Genet. doi:10.1007/s00294-014-0447-9

Medina A, Schmidt-Heydt M, Rodríguez A, Parra R, Geisen R, Magan N (2014) Impacts of environmental stress on growth, secondary metabolite biosynthetic gene clusters and metabolite production of xerotolerant/xerophilic fungi. Curr Genet. doi:10.1007/s00294-014-0455-9

Ortiz-Urquiza A, Keyhani NO (2014) Stress response signaling and virulence: insights from entomopathogenic fungi. Curr Genet. doi:10.1007/s00294-014-0439-9

Rangel DEN, Braga GUL, Anderson AJ, Roberts DW (2005) Variability in conidial thermotolerance of Metarhizium anisopliae isolates from different geographic origins. J Invertebr Pathol $88: 116-125$

Rangel DEN, Anderson AJ, Roberts DW (2006a) Growth of Metarhizium anisopliae on non-preferred carbon sources yields conidia with increased UV-B tolerance. J Invertebr Pathol 93:127-134

Rangel DEN, Butler MJ, Torabinejad J, Anderson AJ, Braga GUL, Day AW, Roberts DW (2006b) Mutants and isolates of 
Metarhizium anisopliae are diverse in their relationships between conidial pigmentation and stress tolerance. J Invertebr Pathol 93:170-182

Rangel DEN, Alston DG, Roberts DW (2008a) Effects of physical and nutritional stress conditions during mycelial growth on conidial germination speed, adhesion to host cuticle, and virulence of Metarhizium anisopliae, an entomopathogenic fungus. Mycol Res 112:1355-1361

Rangel DEN, Anderson AJ, Roberts DW (2008b) Evaluating physical and nutritional stress during mycelial growth as inducers of tolerance to heat and UV-B radiation in Metarhizium anisopliae conidia. Mycol Res 112:1362-1372

Rangel DEN, Fernandes EKK, Dettenmaier SJ, Roberts DW (2010) Thermotolerance of germlings and mycelium of the insect-pathogenic fungus Metarhizium spp. and mycelial recovery after heat stress. J Basic Microb 50:344-350

Rangel DEN, Braga GUL, Fernandes EKK, Keyser CA, Hallsworth JE, Roberts DW (2015) Stress tolerance and virulence of insect-pathogenic fungi are determined by environmental conditions during conidial formation. Curr Genet. doi:10.1007/ s00294-015-0477-y
Selbmann L, Zucconi L, Isola D, Onofri S (2014) Rock black fungi: excellence in the extremes, from the Antarctic to space. Curr Genet. doi:10.1007/s00294-014-0457-7

Shalaby S, Horwitz BA (2014) Plant phenolic compounds and oxidative stress: integrated signals in fungal-plant interactions. Curr Genet. doi:10.1007/s00294-014-0458-6

Solé C, Nadal-Ribelles M, de Nadal E, Posas F (2014) A novel role for lncRNAs in cell cycle control during stress adaptation. Curr Genet. doi:10.1007/s00294-014-0453-y

Williams JP, Hallsworth JE (2009) Limits of life in hostile environments: no barriers to biosphere function? Environ Microbiol 11:3292-3308. doi:10.1111/j.1462-2920.2009.02079.x

Yakimov MM, La Cono V, Spada GL, Bortoluzzi G, Messina E, Smedile F, Arcadi E, Borghini M, Ferrer M, Schmitt-Kopplin P, Hertkorn N, Cray JA, Hallsworth JE, Golyshin PN, Giuliano L (2015) Microbial community of the deep-sea brine Lake Kryos seawater-brine interface is active below the chaotropicity limit of life as revealed by recovery of mRNA. Environ Microbiol 17:364-382. doi:10.1111/1462-2920.12587 